\title{
Antineoplastic-related cardiotoxicity, morphofunctional aspects in a murine model: contribution of the new tool 2D-speckle tracking
}

\author{
Carmela Coppola' \\ Gennaro Riccio' \\ Antonio Barbieri ${ }^{2}$ \\ Maria Gaia Monti ${ }^{3}$ \\ Giovanna Piscopo' \\ Domenica Rea ${ }^{2}$ \\ Claudio Arra ${ }^{2}$ \\ Carlo Maurea' \\ Claudia De Lorenzo ${ }^{4,5}$ \\ Nicola Maurea' \\ 'Division of Cardiology, Istituto \\ Nazionale per lo Studio e la Cura \\ dei Tumori "Fondazione G. Pascale", \\ IRCCS, Naples, Italy; ${ }^{2}$ Animal Facility \\ Unit, Department of Experimental \\ Oncology, Istituto Nazionale per \\ lo Studio e la Cura dei Tumori \\ "Fondazione G. Pascale", IRCCS, \\ Naples, Italy; ${ }^{3}$ Department of \\ Translational Medical Sciences, \\ University Federico II, Naples, Italy; \\ ${ }^{4}$ Department of Molecular Medicine \\ and Medical Biotechnology, University \\ Federico II, Naples, Italy; ${ }^{5}$ CEINGE \\ Biotecnologie Avanzate, Naples, Italy
}

Correspondence: Nicola Maurea Division of Cardiology, Istituto Nazionale per lo Studio e la Cura dei Tumori "Fondazione G. Pascale", IRCCS, 80।3। Naples, Italy

Tel +3908I 5903519

Fax +390815903829

Email n.maurea@istitutotumori.na.it
This article was published in the following Dove Press journal:

OncoTargets and Therapy

2 November 2016

Number of times this article has been viewed

Objective: Considering that global left ventricular systolic radial strain is a sensitive technique for the early detection of left ventricular dysfunction due to antineoplastics and the analysis of segmental myocardial contractility, we evaluated this technique for early detection of trastuzumab-related cardiotoxicity by comparing it with cardiac structural damage.

Methods: Groups of six mice were injected with trastuzumab or doxorubicin, used either as single agents or in combination. Cardiac function was evaluated by transthoracic echocardiography measurements before and after treatment for 2 or 7 days, by using a Vevo 2100 high-resolution imaging system. After echocardiography, mice were euthanized, and hearts were processed for histological evaluations, such as cardiac fibrosis, apoptosis, capillary density, and inflammatory response.

Results: Trastuzumab-related cardiotoxicity was detected early by $2 \mathrm{D}$ strain imaging. Radial strain was reduced after 2 days in mice treated with trastuzumab alone $(21.2 \% \pm 8.0 \%$ vs $40.5 \% \pm 4.8 \%$ sham; $P<0.01$ ). Similarly, trastuzumab was found to induce apoptosis, capillary density reduction, and inflammatory response in cardiac tissue after 2 days of treatment, in a fashion similar to doxorubicin. On the contrary, fractional shortening reduction and cardiac fibrosis were observed only after 7 days of trastuzumab treatment, in contrast to doxorubicin treatment which induced early fibrosis and fractional shortening reduction.

Conclusion: The reduction of left ventricular systolic strain after 2 days of trastuzumab treatment may indicate early myocardial functional damage before the reduction in left ventricular ejection fraction and this early dysfunction is well correlated with structural myocardial damage, such as apoptosis and inflammatory response. Fractional shortening reduction after 7 days of trastuzumab treatment is related to fibrosis in cardiac tissue.

Keywords: trastuzumab, breast cancer, cardiotoxicity, ErbB2/Her2, doxorubicin

\section{Introduction}

The overall survival of cancer patients has been increased by novel therapeutic regimens and the introduction of biological drugs. As a paradox in cancer survivors, cardiotoxicity has emerged as important issue. ${ }^{1}$

Anthracyclines are effective antineoplastic agents in cancer treatment but their clinical use is compromised by the development of a severe form of cardiomyopathy and heart failure (HF). ${ }^{2,3}$ Doxorubicin represents the most effective anthracycline, and the mechanisms of doxorubicin-related cardiac toxicity have been extensively studied. Two main pathways have been proposed; first, doxorubicin induces cardiotoxicity by reactive oxygen species formation; ${ }^{4}$ second, doxorubicin-top $2 \beta$ complex in cardiomyocytes plays a key role to induce changes in mitochondrial membrane potential, function, and morphology. ${ }^{5}$ 
However, also biological agents, such as tyrosine kinase inhibitors and monoclonal antibodies (mAbs) developed to target specific growth signaling pathways, ${ }^{6}$ surprisingly present important side effects due to both "on target" and "off target" activities, ${ }^{7}$ and they can both be associated with severe cardiovascular toxicity. ${ }^{8}$

The cardiotoxicity of anti-ErbB2 antibodies has been initially evidenced for trastuzumab, a humanized anti-ErbB2 monoclonal antibody. ${ }^{9}$ Clinical studies demonstrated that adjuvant trastuzumab administered either after, or in combination with, chemotherapy reduced the risk of relapse by approximately $50 \%$ and the risk of death by $33 \%$ for women with HER-2 positive early breast cancer. ${ }^{10}$ However, cardiac toxicity emerged as an important side effect at an early stage in the development of trastuzumab, manifested as symptomatic congestive $\mathrm{HF}$ or asymptomatic left ventricular ejection fraction (LVEF) decline ${ }^{11}$ that in some cases can cause severe cardiac insufficiency and even death. ${ }^{12}$ Trastuzumab-related cardiotoxicity is due to the inhibition of ErbB4/ErbB2 heterodimerization, ${ }^{13}$ thus leading to the blockage of the NRG activated cardiomyocyte survival pathway. ${ }^{14}$

Initially, two categories of cancer therapy-related cardiotoxicity have been proposed by Ewer and Ewer: Type I and Type II cardiotoxicity. Type I cardiotoxicity, defined as permanent cardiotoxicity, is usually associated with anthracyclines. Type II cardiotoxicity, considered as reversible cardiotoxicity, not associated with histologic alteration, has been mainly related to trastuzumab and other targeted cancer therapy. ${ }^{15}$ Guarnieri et $\mathrm{al}^{16}$ reported that in three patients who experienced a cardiac event (defined by Seidman et al's criteria) $)^{17}$ there was no evidence of structural damage on light microscopy analysis; however, an additional ultrastructural evaluation showed evidence of focal vacuolar changes, pleomorphic mitochondria, myocardial cell hypertrophy, and mild interstitial fibrosis. Anyway, the authors still consider these ultrastructural changes as a reversible type of cardiomyopathy. ${ }^{16}$ Although the number of patients was small and light microscopy is not sensitive enough to detect myocardial damage.

Recently, the cardiotoxic side effects of trastuzumab have been reconsidered, ${ }^{18,19}$ as they can persist many years after the conclusion of the therapy or they can appear many years after the treatment as it takes a long time for the initial heart damage to become clinically evident as $\mathrm{HF},{ }^{18}$ thus strongly suggesting that they are not always reversible as it was initially proposed.

Early detection of this phenomenon could allow for the reversal of this natural progression through an appropriate pharmacological intervention and reconsideration of treatment regimens in oncology. Currently, the two-dimensional echocardiographic assessment of left ventricular (LV) function by calculating the ejection fraction (EF) is the most commonly used methodology in clinical practice for the study of systolic function in patients undergoing antineoplastic treatment. However, this technique, which measures the global myocardial function, has limitations both in the evaluation of segmental myocardial change ${ }^{20}$ and in the early detection of myocardial damage by antineoplastic agents.

The recent development of myocardial strain imaging provides a sensitive and reproducible measurement of LV systolic function.

Plana et al, ${ }^{21}$ in their proposed guidelines, indicated that myocardial deformation can be measured by using Doppler tissue imaging (DTI) or 2D Speckle tracking echocardiography (STE). 2D STE is preferable because it lacks angle dependency. Moreover, Global longitudinal strain (GLS) represents an optimal parameter of deformation for early detection of subclinical LV dysfunction.

The purpose of this study is to compare the alterations of LVEF and LV systolic strain measured with 2D strain imaging after cancer treatment with doxorubicin and the antiErbB2 agent trastuzumab in a mouse model, and to correlate these with histological damage. Global LV systolic radial strain (RS) is a sensitive technique for the early detection of LV dysfunction from antineoplastics, and completes the information obtained with indices of global function. Indeed we have shown that there is a clear correlation between early detection through global LV systolic RS and myocardium damage in trastuzumab-treated mice.

\section{Materials and methods Animal studies}

Seventy two six to eight-week-old female C57Bl/6 mice were purchased by Harlan, San Pietro al Natisone, (Italy). Mice were housed 6 per cage and maintained on a $12 \mathrm{~h}$ light: $12 \mathrm{~h}$ dark cycle (lights on at $7.00 \mathrm{am}$ ) in a temperature-controlled room $\left(22 \pm 2^{\circ} \mathrm{C}\right)$ and with food and water ad libitum at all times. The experimental protocols were in accordance with EU Directive 2010/63/EU for animal experiments, and institutional guidelines of the Italian Ministry of Health Animal Care and Use Committee all animal experiments were approved by the local ethics committees Organismo preposto al benessere degli animali (OPBA).

After 1 week, mice were randomized for weight. All mice were enrolled in treatment groups: Ctr treated with $100 \mu \mathrm{di}$ Saline solution, Doxorubicin $2.17 \mathrm{mg} / \mathrm{Kg} / \mathrm{day}$ as a positive control, Trastuzumab $2.25 \mathrm{mg} / \mathrm{Kg} /$ day (Genentech, South San Francisco, CA, USA), and Doxorubicin plus Trastuzumab via intraperitoneal. 


\section{Transthoracic echocardiography}

In vivo cardiac function was assessed by transthoracic echocardiography in sedated mice by using a Vevo 2100 high-resolution imaging system $(40-\mathrm{MHz}$ transducer; Visualsonics, Toronto, ON, Canada). Mice were anesthetized with tiletamine $(0.09 \mathrm{mg} / \mathrm{g})$, zolazepam $(0.09 \mathrm{mg} / \mathrm{g})$, and $0.01 \%$ atropine $(0.04 \mathrm{~mL} / \mathrm{g})$. Cardiac function was evaluated by noninvasive echocardiography in basal conditions and after treatment (T0-T2-T7) with doxorubicin, trastuzumab, and doxorubicin plus trastuzumab. The LV echocardiogram (ECG) was assessed in both parasternal long-axis and shortaxis views at a frame rate of $233 \mathrm{~Hz}$. End-systole and enddiastole dimensions were defined as the phases corresponding to the ECG T wave, and to the R wave, respectively. M-mode LV end-systolic dimensions (LVESDs) and LV end-diastolic dimensions (LVEDDs) were averaged from 3 to 5 beats. LVEDD and LVESD were measured from the LV M-mode at the mid-papillary muscle level. Fractional shortening percentage $(\% \mathrm{FS})$ was calculated as:

\section{$\left(\right.$ LVEDD-LVESD)/LVEDD) $\times 100 .{ }^{22}$}

RS was evaluated by 2D speckle tracking echocardiography; pictures were captured with a frame rate between 50 e 70 frames/sec and three cardiac cycles were analysed. For strain analysis, endocardial border was traced manually and the myocardial region of interest was automatically identified by the Vevo strain. The curves were analyzed after the selection of the region of interest. The evaluations were performed on the six segments of the short-axis projection standard. Means were calculated automatically by Vevo strain software.

\section{Cardiac fibrosis analysis}

For ex vivo analyses, animals were euthanized after the treatment by cervical dislocation following anesthesia performed as described earlier, the hearts were excised and fixed in $10 \%$ neutral buffered solution.

The myocardial tissue was formalin-fixed and paraffinembedded for morphometry and immunohistochemistry. General morphology was studied with hematoxylin-eosin staining. Six $\mu \mathrm{m}$ thick cross sections were deparaffinized and stained with Picrosirius red (Carlo Erba Laboratories, Milan, Italy) to measure the collagen content. Collagen volume fraction was expressed as the mean percentage of Picrosirius red stained tissue areas divided by total tissue area in the same field and was evaluated in 15 fields at $60 \times$ magnification. ${ }^{23}$ The positively stained (red) fibrotic area was measured by using a computer-assisted image analysis system (Nikon NIS
ELEMENTS BRV; Nikon Instruments, Melville, NY, USA). For measures of capillary density, sections were incubated overnight with biotinylated Bandeiraea simplicifolia Isolectin-I (Sigma-Aldrich Co., St Louis, MO, USA) followed by tyramide signal amplification enhancement (PerkinElmer Inc., Waltham, MA, USA). Capillaries were visualized by DAB, counted and expressed as the number of capillaries per $\mathrm{mm}^{2} .^{24}$

\section{Immunohistochemistry}

Deparaffinized slides were treated with $3 \%$ hydrogen peroxide, nonspecific binding sites were blocked by incubation for 30 minutes with goat blocking serum, followed by overnight incubation with anti-tumor necrosis factor- $\alpha(1: 100)$ or anti CD68 (1:100). ${ }^{24}$ The visualization was performed by ABC kit and DAB chromogenic as peroxidase substrate, and the sections were counterstained with hematoxylin (all products from Thermo Fisher Scientific, Waltham, MA, USA). The percentage of immunopositive cells per total cell number in the same field was calculated and averaged on six sections for myocardial tissue. Morphometric analysis was performed on ten histological sections by using a Nikon Eclipse 1000 microscope equipped with $60 \times$ objective (Nikon Instruments) and image analysis software (Nikon NIS Elements BR) with a digital camera. Measurements were performed independently by two blinded researchers.

\section{Apoptosis}

DNA fragmentation test was performed by using the terminal deoxynucleotidyl transferase mediated dUTP nick end labeling assay (Dead-End Colorimetric TUNEL system; Promega Corporation, Fitchburg, WI, USA) followed by a cytoplasmatic counterstain with eosin. Apoptotic index was calculated as previously described. ${ }^{25}$

\section{Statistics}

Values are indicated as mean \pm standard deviation (SD). Two-way ANOVA and Bonferroni post-hoc analysis were used to examine the significance of differences among groups (Graph and Prism 5.0; GraphPad Software, Inc., La Jolla, CA, USA). A probability value with $P \leq 0.05$ was considered to be statistically significant.

\section{Results}

\section{In vivo cardiotoxic effects and segment analysis}

In order to study the cardiotoxic effects of doxorubicin and trastuzumab, we assessed the effects of these drugs on segmental and global contractility myocardial alterations. Groups of six mice were injected with equimolar doses of 
trastuzumab or doxorubicin, used either as single agents or in combination. Echocardiography measurements were performed on mouse hearts before or after 2 or 7 days treatment. In mice treated with doxorubicin alone, $\mathrm{FS}$ is reduced early (after 2 days) to $56.7 \% \pm 2.4 \%$ vs $61.5 \% \pm 1.2 \%(P<0.001)$ (Figure 1A). Moreover, a combinatorial treatment (doxorubicin plus trastuzumab) induces a drastic reduction of FS in treated mice $(49 \% \pm 2 \%$ vs $61.5 \% \pm 1.2 \%$ in sham; $P<0.001)$. On the contrary, in mice treated with trastuzumab alone, FS decreased after 7 days of treatment $(49 \% \pm 3.0 \%$ vs $60.0 \% \pm 1.0 \%, P<0.001$ ) (Figure 1B).

\section{A}

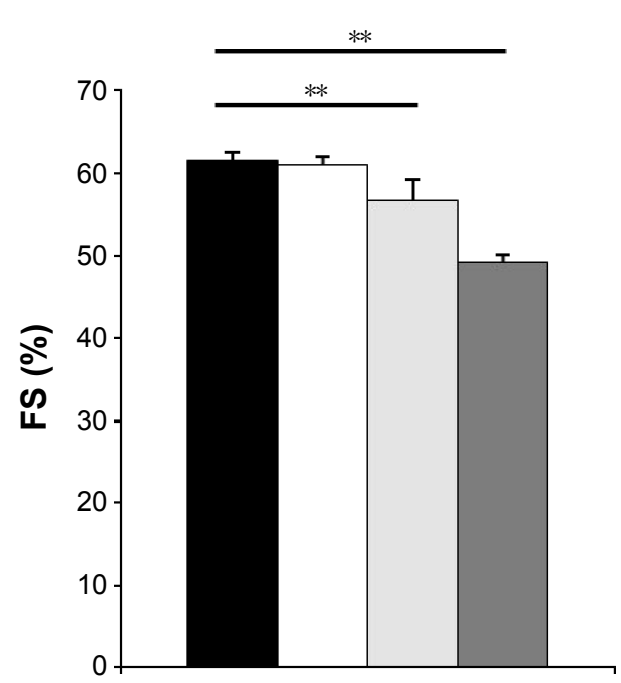

Myocardial strain has shown different results with respect to FS. Indeed RS was already reduced after 2 days in mice treated with doxorubicin alone, with trastuzumab alone (21.2\% $0.0 \%$ vs $40.5 \% \pm 4.8 \%$ sham; $P<0.01)$, and in mice treated with doxorubicin plus trastuzumab. The latter treatment induced a drastic reduction of RS $(14.6 \% \pm 0.8 \%$ vs $41.0 \% \pm 2.8 \%$ in sham, $P=0.001$ ) evaluated by speckle tracking. Thus, speckle tracking was able to identify the cardiotoxicity at a very early stage after only 2 days of treatment. All these data indicate that RS is an early predictor of cardiac dysfunction.

\section{2 days}

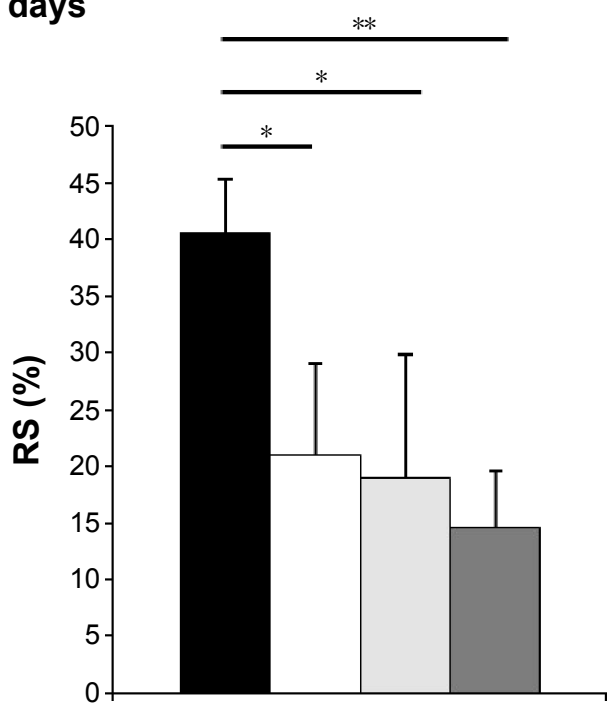

B

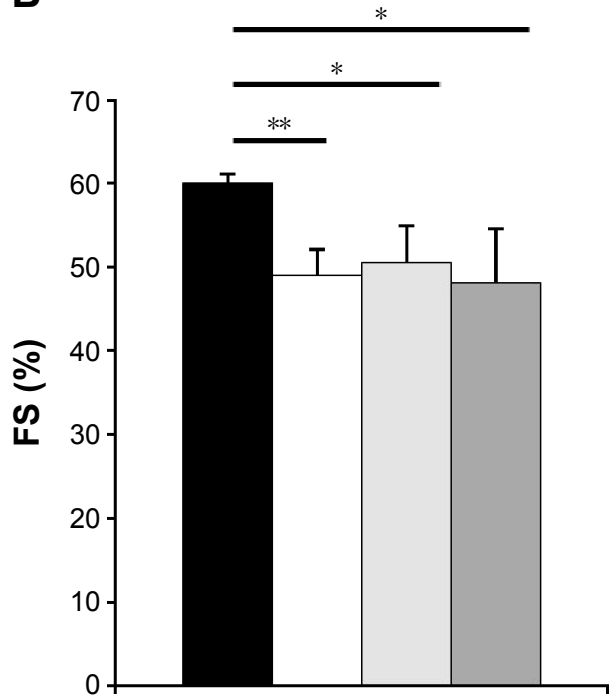

\section{7 days}

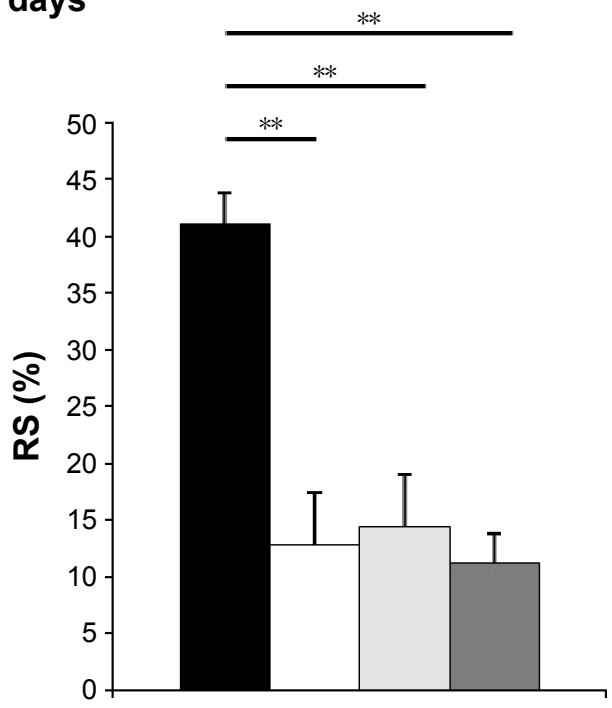

Sham $\square$ Trastuzumab $\square$ Doxorubicin

Doxorubicin plus trastuzumab

Figure I In vivo effects of trastuzumab and doxorubicin.

Notes: Relative FS (fractional shortening) and RS (radial strain), are reported before and after treatment of mice for 2 or 7 days with trastuzumab or doxorubicin used as single agents or in combination. (A) Effects on FS and RS after 2 days of treatment. (B) Effects on FS and RS after 7 days of treatment. $* P<0.0$ I; $* * P<0.00 I$. $P$-values were obtained by two-way ANOVA and Bonferroni post-hoc analysis and error bars are SD. 


\section{Doxorubicin treatment induces reduction of capillary density, apoptosis, and fibrosis}

In order to evaluate the effects of antineoplastic drugs in myocardial tissue, after treatment and echocardiography examination, mice were euthanized and hearts were removed and processed for histological examination. We evaluated cardiac fibrosis after 2 days of treatment by Sirius red staining and apoptosis with TUNEL assay. Capillary density was evaluated by incubating tissue sections with biotinylated Bandeiraea simplicifolia Isolectin-I. As shown in Figure 2, doxorubicin treatment after 2 days induces a drastic reduction of capillary density, an increase in cardiac fibrosis, and increase of the density of apoptotic nuclei in cardiac tissue. Interestingly, the presence of fibrosis after only 2 days correlates with early alteration of FS. Cardiomyocytes showed disomogeneous cytoplasms and damaged disconnected myofibers. In myocardial tissue the distance between cells in treated mice compared to control mice was increased (Figure 2A).

\section{Trastuzumab induces LV dysfunction detected early by RS}

We have shown that treatment with trastuzumab induces apoptosis and a reduction of capillary density, in a fashion similar to doxorubicin treatment, whereas the cardiac fibrosis becomes important and statistically significant after 7 days of treatment which correlates with an important alteration of FS (Figure 2C). In trastuzumab-treated mice, the hearts showed reactive fibrosis limited to the perivascular areas. Moreover, we observed a few deposits of collagen "star" between cardiomyocytes. Interestingly, cardiac damage after 2 days of trastuzumab treatment was identified early with alteration of RS, which correlates histologically with an increased apoptotic index in cardiac samples (Figure 2B). The cytoplasm of cardiomyocytes results homogeneous, the nuclei have a normal appearance and no space or vacuoles are present (Figure 2A). These results suggest that trastuzumab is able to induce histological alteration in cardiac tissue of treated mice, thus leading to functional alterations detected early by RS.

\section{Doxorubicin and trastuzumab treatment induces inflammatory response in cardiac tissue}

Inflammatory response was evaluated by immunohistochemistry analysis with anti-TNF- $\alpha$ and anti-CD68 mAbs. An increase in inflammatory response has been observed, caused by trastuzumab and doxorubicin treatment, indicated by overexpression of TNF- $\alpha$ in cardiac tissue (Figure 3), which is an inflammatory cytokine mainly secreted by macrophages. Moreover, in cardiac tissue of treated mice, it is possible to also observe an increased presence of macrophages, as indicated by positive staining with anti-CD68 mAb (Figure 4). The cellular infiltrates in trastuzumab treatment are more abundant with respect to the other treatments, and the distribution of immunostaining for anti-TNF- $\alpha$ is uneven.

This result can be explained by the fact that during the apoptotic process dying cells display molecules that mark them for phagocytosis, thus attracting macrophages into cardiac tissues. The presence of an inflammatory response indicates early damage due to doxorubicin and trastuzumab treatment.

\section{Discussion}

In recent years, the use of potentially cardiotoxic antineoplastic drugs has improved the survival of cancer patients, but it has actually created a new category of individuals at risk of HF. In $50 \%$ of cases, the HF is preceded, even for years, by asymptomatic left ventricular dysfunction (LVSD). LVSD itself, uncorrelated to the degree of impairment of ventricular function and future development of HF, increases the risk of death. ${ }^{11}$ When LVSD evolves in HF, the prognosis is unfavorable and it is comparable to that of most neoplasia: $50 \%$ die within 5 years from diagnosis and 10 -year survival is $20 \%$. Anthracyclines represent highly potent antineoplastic agents used against hematological and solid tumors. However, their use has been limited by cardiotoxic adverse effects, ${ }^{3}$ which may lead to congestive HF. The cardiac toxicity is directly related to the cumulative dose of anthracyclines received. However, microscopic analysis revealed myocardial damage already at doses as low as $180 \mathrm{mg} / \mathrm{m}^{2}$. The anthracyclinerelated cardiotoxicity could be a problem even years after the last administration. Indeed, ventricular remodeling triggered by myocardial damage as a consequence of a single low dose of anthracyclines is an ongoing process and it can take a long time from initial injury to clinically overt HF. ${ }^{20}$ Trastuzumab is a humanized antibody specific for receptor tyrosine kinase ErbB2, overexpressed in $25 \%$ of cases of breast cancer. ErbB2 expression in tumors is associated with a poor disease-free patient survival. ${ }^{26}$ One of the most significant forms of cardiotoxicity associated with trastuzumab affects the myocardium, decreasing LVEF, which can then progress to $\mathrm{HF}$ and in some cases death. ${ }^{11}$

The incidence of cardiac dysfunction is higher in patients who have had prior anthracycline treatment, a known trigger for myocyte death, suggesting that inhibition of cardiac 

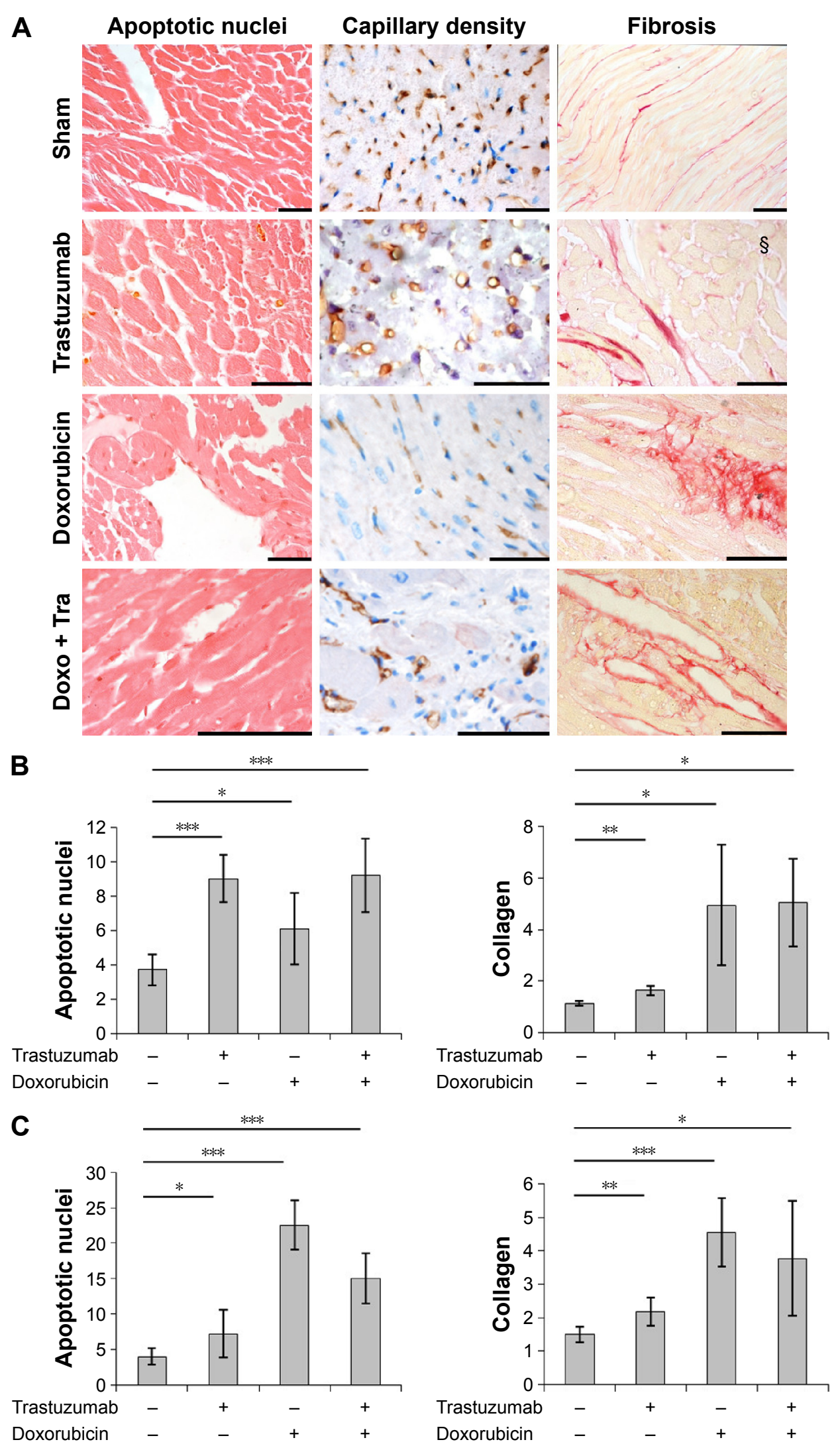

Figure 2 Effects on cardiac tissue of trastuzumab (Tra) and doxorubicin (Doxo).

Notes: (A) Representative photomicrographs of LV (left ventricular) sections from mice treated for 2 days with the indicated drugs; ffibrosis in cardiac tissue of trastuzumabtreated mice is observed after only 7 days. (B, C) Quantification of the apoptotic nuclei and interstitial fibrosis reported as relative percentage of positive nuclei and Picrosirius red stained fibers after 2 days $(\mathbf{B})$ and after 7 days $(\mathbf{C})$. ${ }^{* P}<0.05 ; * * P<0.01 ; * * * P<0.00$ I. P-values were obtained by two-way ANOVA and Bonferroni post-hoc analysis and error bars are SD. Scale bar $=50 \mu \mathrm{m}$. 
A

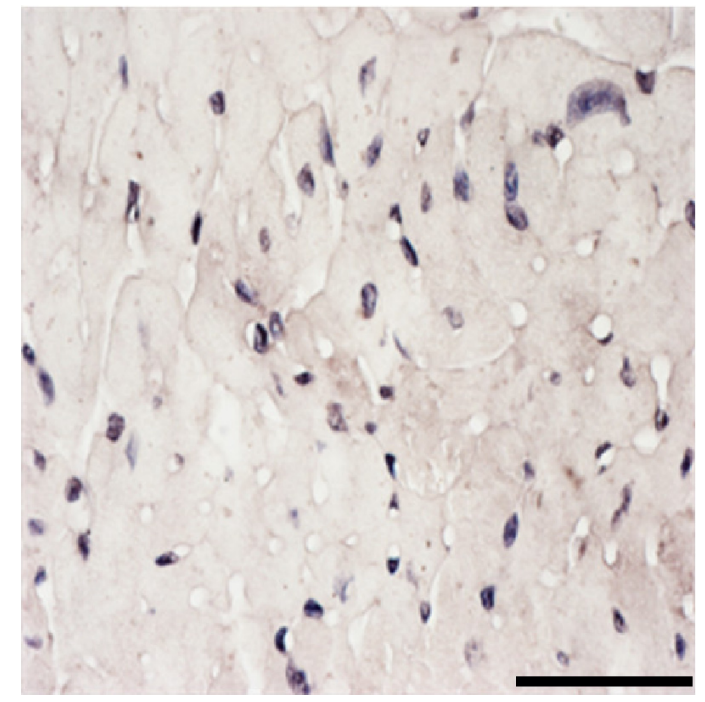

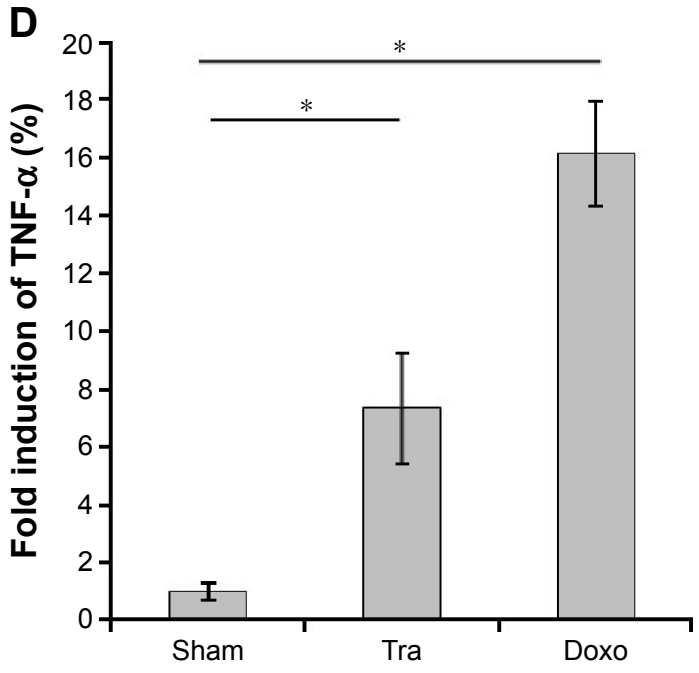

B

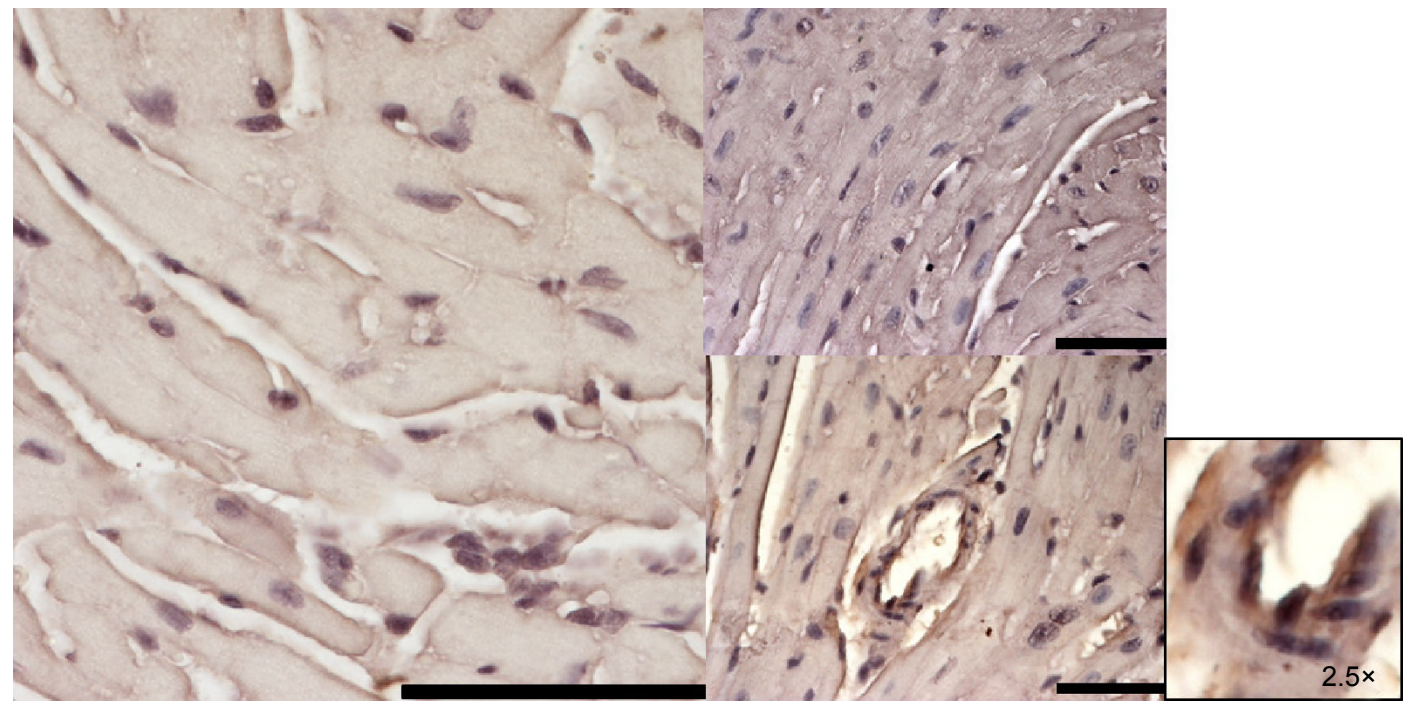

C
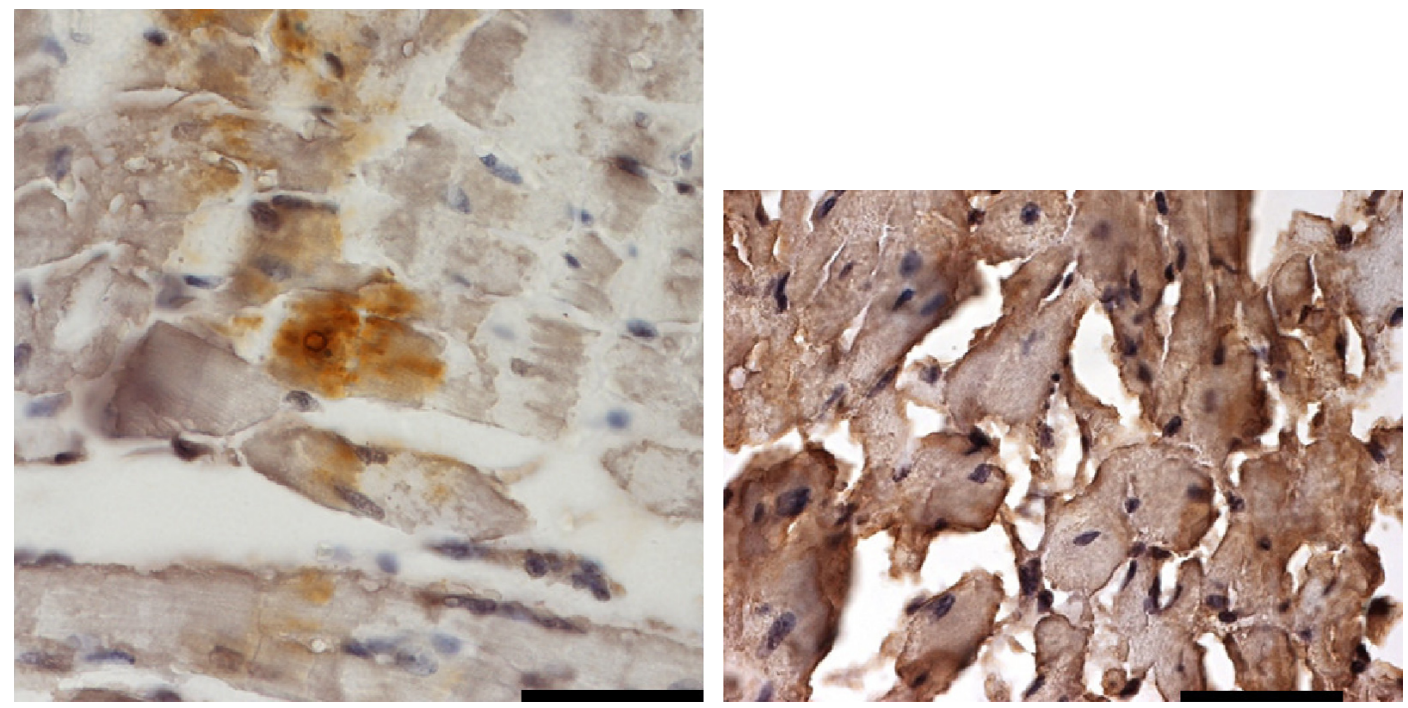

Figure 3 Analysis of TNF- $\alpha$ in cardiac tissue of treated mice.

Notes: (A) Cardiac tissue of untreated mice. (B) Cardiac tissue of trastuzumab-treated mice. (C) Cardiac tissue of doxorubicin-treated mice. (D) Quantification of the TNF- $\alpha$ expression reported as relative percentage, after 2 days of treatment. $* P<0.0$ I. $P$-values were obtained by two-way ANOVA and Bonferroni post-hoc analysis and error bars are SD. Scale bar $=50 \mu \mathrm{m}$.

Abbreviations: TNF- $\alpha$, tumor necrosis factor; Tra, trastuzumab; Doxo, doxorubicin. 

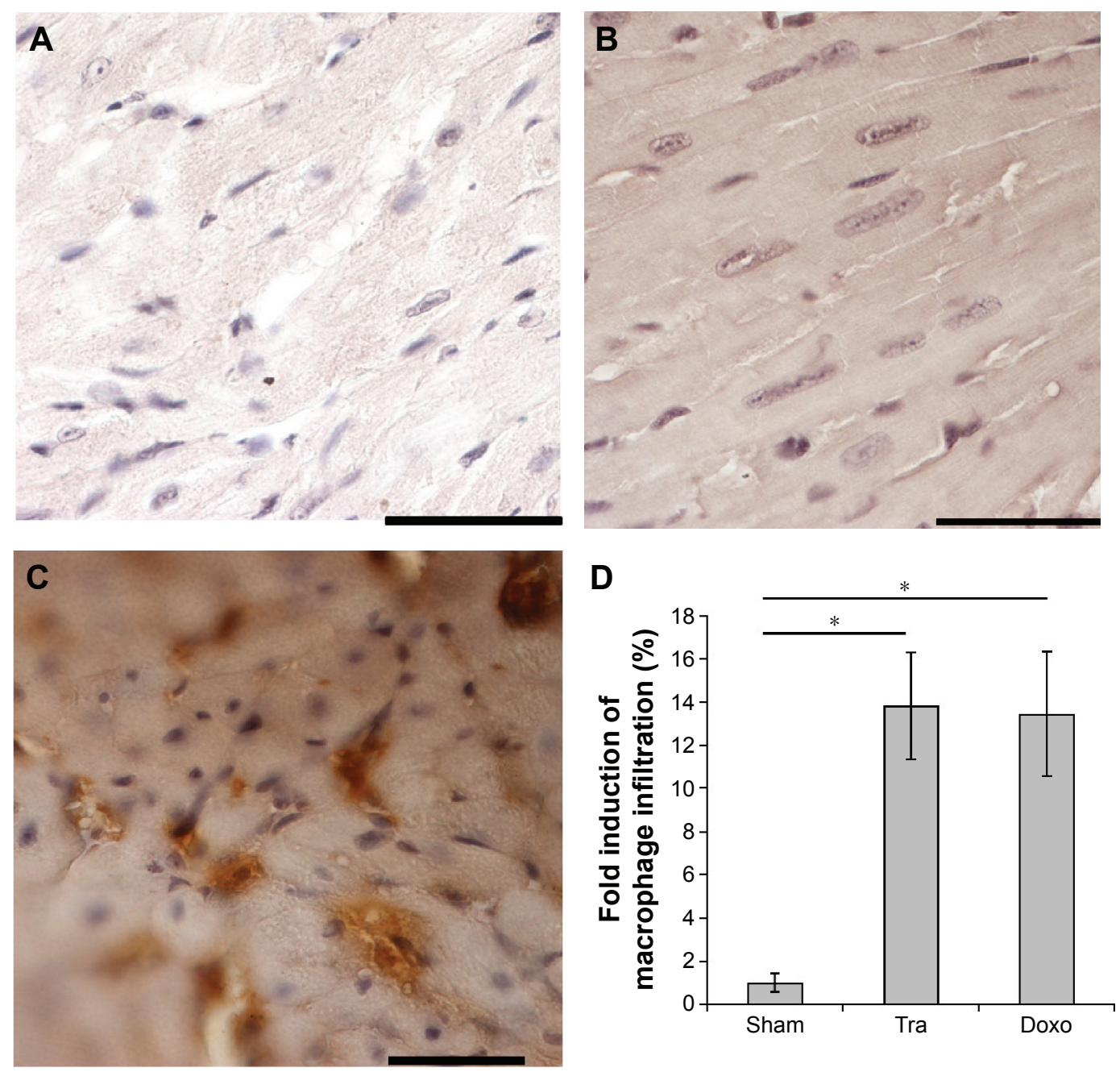

Figure 4 Analysis of macrophage infiltration in cardiac tissues of treated mice by staining with anti-CD68.

Notes: (A) Cardiac tissue of untreated mice. (B) Cardiac tissue of trastuzumab-treated mice. (C) Cardiac tissue of doxorubicin-treated mice. (D) Quantification of the CD68 staining reported as relative percentage, after 2 days of treatment. $* P<0.01$. $P$-values were obtained by two-way ANOVA and Bonferroni post-hoc analysis and error bars are SD. Scale bar $=50 \mu \mathrm{m}$.

Abbreviations: Tra, trastuzumab; Doxo, doxorubicin.

repair mechanisms is a clinically relevant determinant of LVSD. ${ }^{27}$ Thus, receptor blockade by trastuzumab prevents this protection mechanism that is triggered by anthracycline treatment or other comorbidity. ${ }^{28}$

The determination of biomarkers in this setting has shown some limitations..$^{29}$ Traditional echocardiographic index of cardiac functionality does not identify early damage induced by anticancer drugs. In this work, we evaluated whether myocardial strain through 2D-speckle tracking is useful to detect early myocardial dysfunction in a mouse model of cardiotoxicity due to doxorubicin and trastuzumab. A very interesting study ${ }^{30}$ has recently indicated changes in LV diastolic function using 2D STE immediately after administration of therapeutic doses of anthracycline chemotherapy. Data analysis indicates that the changes in diastolic function are related to reduced systolic function. Reduced longitudinal strain with preserved LVEF has been previously reported in other disease settings. ${ }^{31-33} \mathrm{~A}$ reduction in radial 2D strain has been reported in an animal model early after anthracycline therapy. ${ }^{34}$ Stoodley et $\mathrm{al}^{20}$ reported that 2D myocardial strain imaging detects changes in LV systolic function immediately after anthracyclines. Twodimensional strain is a new simple, rapid, and reproducible method to measure different components of systolic strain. ${ }^{35,36}$ This technique identified early abnormalities in patients with potential cardiotoxicity even though they had apparently normal LV systolic function. Clinical use of 2D strain has some advantages with respect to TDI strain, which can present some artifacts caused by myocardial translational motion, requirement for optimal Doppler alignment, variable reproducibility, and by time-consuming off-line analysis.

In this study, we report a comparative analysis of doxorubicin-related and anti-ErbB2-related cardiotoxic side 
effects. Cardiotoxic effects were evaluated by traditional echocardiographic index and by 2D-speckle tracking (ST), to study global myocardial function and to perform segmental analysis. Moreover, histological analysis of cardiac tissues was performed to evaluate cardiac damage.

Doxorubicin has been found to induce early myocardial contractility alteration, as shown by FS and RS reduction after 2 days of treatment. This contractile dysfunction is related to high inflammatory response, death of cardiomyocytes by apoptosis, and fibrosis in cardiac tissues. Cellular damage is an ongoing process and after 7 days of treatment the inflammatory and apoptotic index drastically increase.

In trastuzumab-treated mice cardiotoxic effects, detected by conventional echocardiographic technique, appear later with respect to doxorubicin-treated mice. Alterations in LV function are evidenced later (after 7 days) with conventional echocardiographic technique in trastuzumab-treated mice; on the contrary, alterations of RS obtained by 2D-ST are detected earlier (after 2 days of treatment), thus preceding the reduction of traditional index of myocardial contractility (EF and FS). Furthermore, trastuzumab induces early inflammatory response and apoptosis in cardiac tissue, whereas fibrosis was observed after 7 days of treatment, when traditional contractility index also changed. Thus, with traditional echocardiographic technique, trastuzumab-related toxicity is identified after 7 days, when fibrosis is also observed in cardiac tissue, which is an irreversible degenerating process also related to aging. ${ }^{37}$ On the contrary, after only 2 days of treatment we observed functional (RS) and histological variations. Thus, RS alterations anticipate change of EF and FS and have been shown to correlate with inflammatory response and apoptosis, which anticipates fibrosis.

Moreover, these data are in contrast with the classification of Type II trastuzumab-related cardiotoxicity proposed by Ewer and Ewer ${ }^{15}$ that indicates the absence of histological alterations in trastuzumab-related cardiotoxicity after the treatment. Guarnieri et $\mathrm{al}^{16}$ reported ultrastructural damage associated with reversible cardiomyopathy. We have already discussed the classification of antineoplasticrelated cardiotoxicity, ${ }^{38}$ and we should take into account that trastuzumab-related cardiotoxicity, in clinical practice, is not always reversible.

Clinical translation of these experimental results can lead to the use of RS with 2D-ST for early identification of antineoplastic-related cardiotoxicity in oncological patients, thus preventing contractile dysfunction in LV. Moreover, we report that trastuzumab may induce fibrosis and other alterations in cardiac tissue that lead to an irreversible cardiac dysfunction. This may be related to irreversible cardiomyopathy observed in clinical practice.

We may conclude that: 1) trastuzumab induces early structural alterations, such as apoptosis and inflammation in cardiac tissue of treated mice in a fashion similar to doxorubicin. These alterations are related to early RS alteration, subsequently, other structural changes such as fibrosis, and the reduction of traditional cardiotoxicity indexes (FS and EF) have been reported; 2) RS in mice can be measured with good reproducibility and it is significantly reduced only after 2 days of antineoplastic treatment, which then lead to contractile dysfunction.

\section{Disclosure}

The authors report no conflicts of interest in this work.

\section{References}

1. Eschenhagen T, Force T, Ewer MS, et al. Cardiovascular side effects of cancer therapies: a position statement from the Heart Failure Association of the European Society of Cardiology. Eur J Heart Fail. 2011; 13(1):1-10.

2. Khouri MG, Douglas PS, Mackey JR, et al. Cancer therapy-induced cardiac toxicity in early breast cancer: addressing the unresolved issues. Circulation. 2012;126(23):2749-2763.

3. Hershman DL, Shao T. Anthracycline cardiotoxicity after breast cancer treatment. Oncology (Williston Park). 2009;23(3):227-234.

4. Singal PK, Iliskovic N. Doxorubicin-induced cardiomyopathy. N Engl J Med. 1998;339(13):900-905.

5. Zhang S, Liu X, Bawa-Khalfe T, et al. Identification of the molecular basis of doxorubicin-induced cardiotoxicity. Nat Med. 2012;18(11): 1639-1642.

6. Ferrara N, Kerbel RS. Angiogenesis as a therapeutic target. Nature. 2005;438(7070):967-974.

7. Dy GK, Adjei AA. Understanding, recognizing, and managing toxicities of targeted anticancer therapies. CA Cancer J Clin. 2013;63(4): 249-279.

8. Liu S, Kurzrock R. Toxicity of targeted therapy: implications for response and impact of genetic polymorphisms. Cancer Treat Rev. 2014; 40(7):883-891.

9. Stebbing J, Copson E, O'Reilly S. Herceptin (trastuzamab) in advanced breast cancer. Cancer Treat Rev. 2000;26(4):287-290.

10. Romond EH, Perez EA, Bryant J, et al. Trastuzumab plus adjuvant chemotherapy for operable HER2-positive breast cancer. NEngl J Med. 2005;353(16):1673-1684.

11. Tocchetti CG, Ragone G, Coppola C, et al. Detection, monitoring, and management of trastuzumab-induced left ventricular dysfunction: an actual challenge. Eur J Heart Fail. 2012;14(2):130-137.

12. Maurea N, Coppola C, Ragone G, et al. Women survive breast cancer but fall victim to heart failure: the shadows and lights of targeted therapy. J Cardiovasc Med (Hagerstown). 2010;11(12):861-868.

13. Fedele C, Riccio G, Malara AE, D'Alessio G, De Lorenzo C. Mechanisms of cardiotoxicity associated with ErbB2 inhibitors. Breast Cancer Res Treat. 2012;134(2):595-602.

14. Chien KR. Myocyte survival pathways and cardiomyopathy: implications for trastuzumab cardiotoxicity. Semin Oncol. 2000;27(6 Suppl 11): 9-14; discussion 92-100.

15. Ewer SM, Ewer MS. Cardiotoxicity profile of trastuzumab. Drug Saf. 2008;31(6):459-467.

16. Guarneri V, Lenihan DJ, Valero V, et al. Long-term cardiac tolerability of trastuzumab in metastatic breast cancer: the M.D. Anderson Cancer Center experience. J Clin Oncol. 2006;24(25):4107-4115. 
17. Seidman A, Hudis C, Pierri MK, et al. Cardiac dysfunction in the trastuzumab clinical trials experience. J Clin Oncol. 2002;20(5): $1215-1221$.

18. Bowles EJ, Wellman R, Feigelson HS, et al. Risk of heart failure in breast cancer patients after anthracycline and trastuzumab treatment: a retrospective cohort study. J Natl Cancer Inst. 2012;104(17): 1293-1305.

19. Chen J, Long JB, Hurria A, Owusu C, Steingart RM, Gross CP. Incidence of heart failure or cardiomyopathy after adjuvant trastuzumab therapy for breast cancer. J Am Coll Cardiol. 2012;60(24): 2504-2512.

20. Stoodley PW, Richards DA, Hui R, et al. Two-dimensional myocardial strain imaging detects changes in left ventricular systolic function immediately after anthracycline chemotherapy. Eur J Echocardiogr. 2011;12(12):945-952.

21. Plana JC, Galderisi M, Barac A, et al. Expert consensus for multimodality imaging evaluation of adult patients during and after cancer therapy: a report from the American Society of Echocardiography and the European Association of Cardiovascular Imaging. Eur Heart J Cardiovasc Imaging. 2014;15(10):1063-1093.

22. Fedele C, Riccio G, Coppola C, et al. Comparison of preclinical cardiotoxic effects of different ErbB2 inhibitors. Breast Cancer Res Treat. 2012;133(2):511-521.

23. Cittadini A, Napoli R, Monti MG, et al. Metformin prevents the development of chronic heart failure in the SHHF rat model. Diabetes. 2012;61(4):944-953.

24. Cittadini A, Monti MG, Iaccarino G, et al. SOCS1 gene transfer accelerates the transition to heart failure through the inhibition of the gp130/ JAK/STAT pathway. Cardiovasc Res. 2012;96(3):381-390.

25. Kajstura J, Cheng W, Reiss K, et al. Apoptotic and necrotic myocyte cell deaths are independent contributing variables of infarct size in rats. Lab Invest. 1996;74(1):86-107.

26. Ravdin PM, Chamness GC. The c-erbB-2 proto-oncogene as a prognostic and predictive marker in breast cancer: a paradigm for the development of other macromolecular markers - a review. Gene. 1995; 159(1):19-27.

27. Ewer MS, Ewer SM. Cardiotoxicity of anticancer treatments: what the cardiologist needs to know. Nat Rev Cardiol. 2010;7(10):564-575.
28. Serrano C, Cortes J, De Mattos-Arruda L, et al. Trastuzumab-related cardiotoxicity in the elderly: a role for cardiovascular risk factors. Ann Oncol. 2012;23(4):897-902.

29. Tocchetti CG, Coppola C, Arra C, Maurea N. Early identification of left ventricular dysfunction induced by trastuzumab. J Am Coll Cardiol. 2011;58(25):2698-2699; author reply 2699-2700.

30. Stoodley PW, Richards DA, Boyd A, et al. Altered left ventricular longitudinal diastolic function correlates with reduced systolic function immediately after anthracycline chemotherapy. Eur Heart J Cardiovasc Imaging. 2013;14(3):228-234.

31. Koyama J, Davidoff R, Falk RH. Longitudinal myocardial velocity gradient derived from pulsed Doppler tissue imaging in AL amyloidosis: a sensitive indicator of systolic and diastolic dysfunction. J Am Soc Echocardiogr. 2004;17(1):36-44.

32. Serri K, Reant $P$, Lafitte $M$, et al. Global and regional myocardial function quantification by two-dimensional strain: application in hypertrophic cardiomyopathy. J Am Coll Cardiol. 2006;47(6):1175-1181.

33. Weidemann F, Breunig F, Beer M, et al. Improvement of cardiac function during enzyme replacement therapy in patients with Fabry disease: a prospective strain rate imaging study. Circulation. 2003; 108(11):1299-1301.

34. Migrino RQ, Aggarwal D, Konorev E, Brahmbhatt T, Bright M, Kalyanaraman B. Early detection of doxorubicin cardiomyopathy using two-dimensional strain echocardiography. Ultrasound Med Biol. 2008;34(2):208-214.

35. Ho E, Brown A, Barrett P, et al. Subclinical anthracycline- and trastuzumab-induced cardiotoxicity in the long-term follow-up of asymptomatic breast cancer survivors: a speckle tracking echocardiographic study. Heart. 2010;96(9):701-707.

36. Stoodley PW, Richards DA, Meikle SR, Clarke J, Hui R, Thomas L. The potential role of echocardiographic strain imaging for evaluating cardiotoxicity due to cancer therapy. Heart Lung Circ. 2011;20(1):3-9.

37. Biernacka A, Frangogiannis NG. Aging and cardiac fibrosis. Aging Dis. 2011;2(2):158-173.

38. Riccio G, Coppola C, Piscopo G, et al. Trastuzumab and targettherapy side effects: is still valid classification between Anthracyclines Type I and Target Therapy Type II cardiomyopathies? Hum Vaccin Immunother. 2016;12(5):1124-1131.
OncoTargets and Therapy

\section{Publish your work in this journal}

OncoTargets and Therapy is an international, peer-reviewed, open access journal focusing on the pathological basis of all cancers, potential targets for therapy and treatment protocols employed to improve the management of cancer patients. The journal also focuses on the impact of management programs and new therapeutic agents and protocols on

\section{Dovepress}

patient perspectives such as quality of life, adherence and satisfaction The manuscript management system is completely online and includes a very quick and fair peer-review system, which is all easy to use. Visit http://www.dovepress.com/testimonials.php to read real quotes from published authors. 